\title{
Calculation of the Meshing Point Position of Cycloidal-pin Wheel Drive for RV Reducer in Robot
}

\author{
Tianxing $\mathrm{Li}^{1, \mathrm{a}}$, Jingzhao Yang ${ }^{1, \mathrm{~b}}$, Xiaozhong Deng ${ }^{2, \mathrm{c}}$, \\ Guofeng Wang ${ }^{1}$ and Chunrong Xing ${ }^{1}$ \\ ${ }^{1}$ School of Mechatronics Engineering, Henan University of Science \& Technology, \\ Luoyang 471003,China \\ ${ }^{2}$ Collaborative Innovation Center of Machinery Equipment Advanced Manufacturing of \\ Henan Province, Luoyang 471003,China \\ aly_litianxing@163.com, byangjingzhao01@163.com, dxz01@163.com
}

\begin{abstract}
Keywords: RV reducer in robot, Cycloidal-pin wheel, Meshing point position, TCA
Abstract. The purpose of this paper is to ascertain the meshing point position of the cycloidal-pin wheel, which is the basis of studying the transmission performance of RV(Rotate Vector) reducer in robot. In terms of the general theoretical tooth profile equation of cycloid gear in RV reducer, the space meshing motion relationship of cycloidal-pin wheel is analyzed. Finally, the accurate meshing points positions of standard cycloidal-pin wheel and modified cycloidal-pin wheel at any angle under no load are obtained by means of tooth contact analysis(TCA). The results prove the meshing theories of cycloidal-pin wheel drive and provide the theoretical support for the actual meshing performance analysis of cycloidal-pin wheel planetary transmission.
\end{abstract}

\section{Introduction}

The cycloidal-pin wheel is the key component of RV reducer, which is a planetary gear drive with few teeth. Studying on the meshing point position of the cycloidal-pin wheel drive is of great significance to further research on the transmission performance, such as meshing backlash, stress, deformation and transmission error.

At present, experts and scholars have carried on the basic research to the transmission principle of the cycloidal-pin wheel planetary drive in RV reducer. Lixing Li and Chunhe Hong analyzed the principle of cycloid-pin wheel drive and deduced the universal cycloid gear equation[1]. Wan-Sung Lin, Yi-Pei Shih and Jyh-Jone Lee analyzed the meshing transmission performance of cycloidal-pin wheel drive[2].Xiaozhong Deng and Weidong $\mathrm{He}$ et al. researched the development status of the cycloidal-pin wheel in RV reducer and illustrated the domestic technical bottleneck, which had practical guiding significance[3,4]. Dongsheng Zhang and Tianmin Guan based on the theory of dynamic change of the first contact point, obtained the distribution interval of the first contact point [5]. But for the calculation method of the meshing point position of the cycloidal-pin wheel under no-load condition, there is no specific theoretical study to date. Therefore, the meshing point position at any moment under the no-load condition is achieved by means of gear contact analysis(TCA).

\section{Mathematical Modeling of Cycloidal-pin Wheel Meshing Contact}

The cycloidal-pin wheel planetary drive of RV reducer in industrial robot is complicated that could be analyzed by the motion transition model, as shown in Fig. 1. Coordinate system $S_{f}$ is fixed with rigid frame, coordinate system $S_{1}$ and $S_{2}$ are fixed with pin wheel and cycloid gear respectively. $\phi_{1}$ and $\phi_{2}$ are the angles of pin wheel and cycloid gear respectively. The rotation center $O_{1}$ of the pin wheel is coincident with the origin of fixed coordinate system $S_{f} . O_{2}$ is the rotation center of cycloid gear. $a$ is the eccentricity between the cycloid gear and the pin wheel axis. 


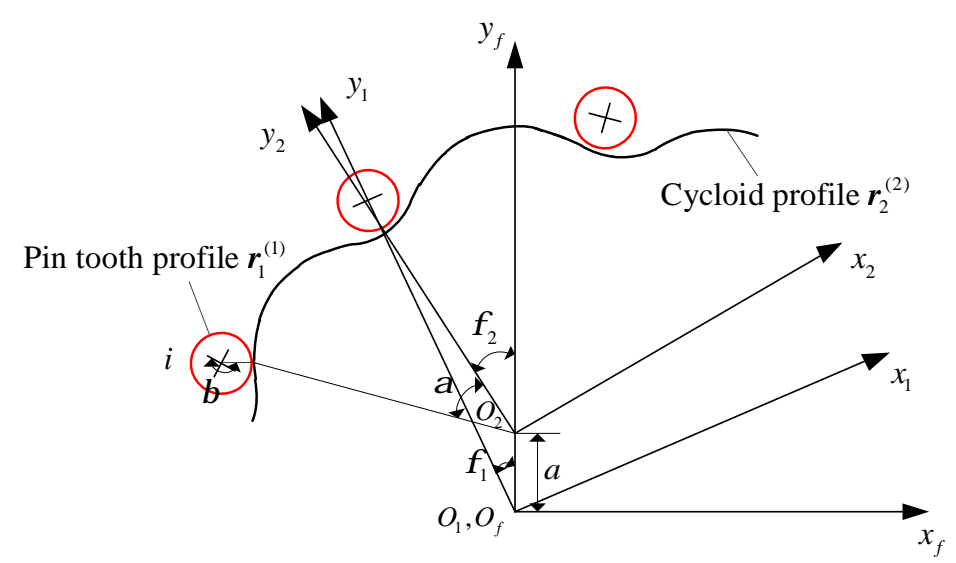

Fig. 1. Cycloidal-pin wheel pair meshing conversion coordinate system

According to gear meshing principle and differential geometry theory, the pin tooth profile $\boldsymbol{r}_{1}^{(1)}$ in the coordinate system $S_{1}$ is

$$
\boldsymbol{r}_{1}^{(1)}=\left[\begin{array}{l}
-r_{r p} \sin \beta \cos \left(\frac{2 \pi n_{i}}{z_{p}}\right)-\sin \left(\frac{2 \pi n_{i}}{z_{p}}\right)\left(r_{r p} \cos \beta+r_{p}\right) \\
-r_{r p} \sin \beta \sin \left(\frac{2 \pi n_{i}}{z_{p}}\right)+\cos \left(\frac{2 \pi n_{i}}{z_{p}}\right)\left(r_{r p} \cos \beta+r_{p}\right) \\
b_{p} \\
1
\end{array}\right]
$$

In Eq. $1, r_{p}$ is the radius of pin wheel, $r_{r p}$ is the radius of pin tooth, $z_{p}$ is the number of pin teeth, $\beta$ is the profile parameter of pin wheel, $n_{i}$ is the $i$-th pin tooth number, $b_{p}$ is width of pin tooth. The cycloid profile $\boldsymbol{r}_{2}^{(2)}$ in the coordinate system $S_{2}$ is derived from document[2].

The coordinate vector of the pin wheel $\boldsymbol{r}_{1}^{(1)}$ and the unit normal vector $\boldsymbol{n}_{1}^{(1)}$ are respectively expressed in $S_{f}$.

$$
\left\{\begin{array}{l}
\boldsymbol{r}_{f}^{(1)}\left(\beta, \phi_{1}\right)=\boldsymbol{M}_{f 1} \boldsymbol{r}_{1}^{(1)} \\
\boldsymbol{n}_{f}^{(1)}\left(\beta, \phi_{1}\right)=\boldsymbol{M}_{f 1} \boldsymbol{n}_{1}^{(1)}
\end{array}\right.
$$

In Eq. 2, $\boldsymbol{M}_{f 1}$ is coordinate transformation matrix from $S_{1}$ to $S_{f} \cdot \boldsymbol{r}_{f}^{(1)}$ and $\boldsymbol{n}_{f}^{(1)}$ are coordinate vector and unit normal vector of the needle wheel in $S_{f}$ respectively. Similarly, the coordinate vector of the cycloid gear $\boldsymbol{r}_{2}^{(2)}$ and the unit normal vector $\boldsymbol{n}_{2}^{(2)}$ are respectively expressed in $S_{f}$.

In the process of cycloidal-pin wheel transmission, the two tooth surface must be in continuous tangency contact. Therefore in the fixed coordinate system $S_{f}$, the position vector of the cycloidal-pin profile at the meshing point is equal to the unit normal vector. The TCA equations are shown in Eq. 3.

$$
\left\{\begin{array}{l}
\boldsymbol{r}_{f}^{(1)}\left(\beta, \phi_{1}\right)=\boldsymbol{r}_{f}^{(2)}\left(\alpha, \phi_{2}\right) \\
\boldsymbol{n}_{f}^{(1)}\left(\beta, \phi_{1}\right)=\boldsymbol{n}_{f}^{(2)}\left(\alpha, \phi_{2}\right)
\end{array}\right.
$$

\section{Meshing Point Position of Standard Cycloidal-pin Wheel}

Standard cycloid profile is continuous cycloid, formed by short epicycloid. The standard cycloid gear and pin tooth profile conjugate everywhere, so the standard cycloidal-pin wheel drive is multi teeth meshing under no load and half of the teeth are engaged in meshing in early theory. In this paper, the accurate meshing point position of cycloidal-pin wheel at any angle is obtained by means of combining TCA with geometric analysis. Fig. 2 is a standard cycloid-pin wheel drive pair in $S_{f} . P$ is a fixed node, which is the intersection point between the cycloid gear pitch and the pin wheel pitch. $M_{i}$ is the center 
point of the $i$-th pin tooth. $k_{i}$ is the meshing point between the $i$-th pin tooth and cycloid wheel. $\theta_{b i}$ is the included angle between $M_{i}$ and $y_{f}$ in $S_{f}, \theta_{b i}=2 \pi n_{i} / z_{p}+\phi_{1} \cdot \theta_{i}$ is an angle parameter, $\theta_{i}=\arccos \left(\frac{{\overline{M_{i} P}}^{2}+{\overline{P O_{1}}}^{2}-r_{p}^{2}}{2 \overline{M_{i} P} \cdot \overline{P O_{1}}}\right), \overline{M_{i} P}=r_{p} \cdot S^{\frac{1}{2}}, \overline{P O_{1}}=a \cdot z_{p}, S=1+k_{1}^{2}-2 k_{1} \cos \theta_{b i}$.

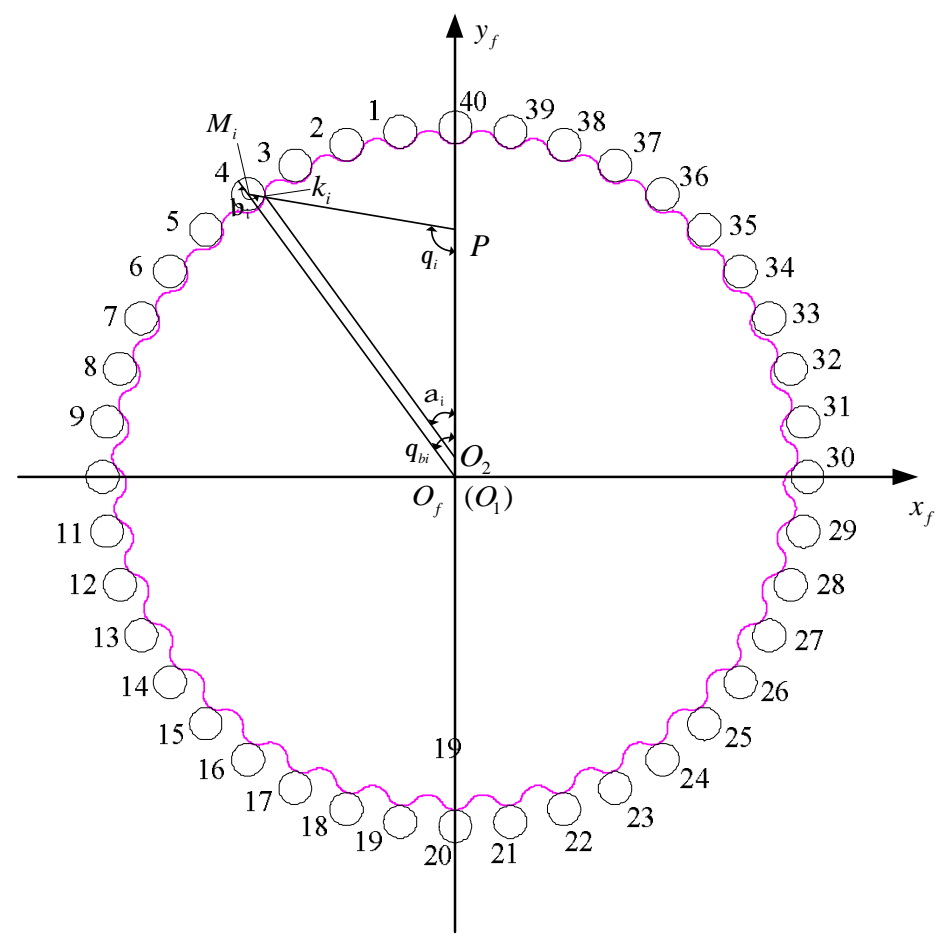

Fig. 2. Standard cycloid-pin wheel drive

A standard cycloidal-pin wheel in RV reducer is taken as an example, all the technical parameters are $z_{p}=40, z_{c}=39, a=1.3 \mathrm{~mm}, r_{p}=64 \mathrm{~mm}, r_{r p}=3 \mathrm{~mm}, \Delta r_{p}=0, \Delta r_{r p}=0, \delta=0$. As shown in Fig. 2, the position of the cycloidal-pin wheel in $S_{f}$ here as the initial position. If pin wheel turn $\phi_{1}$, the position parameters at each meshing point are calculated by the TCA equations(Eq. 3). The meshing points positions of standard cycloidal-pin wheel are shown in Table 1 . Pin wheel $\phi_{1}=0.4 \mathrm{rad}$, then cycloid gear is $\phi_{2}=0.4103 \mathrm{rad}$, the parameters of each meshing point $\alpha_{i}$ and $\beta_{i}$ are obtained.The results are accurate because of the maximum error $\Delta_{\max }=1.99 \times 10^{-11}$. The conclusion proves the theory that all pairs of teeth would mesh in the transmission process of standard cycloidal-pin wheel at any moment under no load.

Table 1 Meshing points positions of standard cycloidal-pin wheel $\left(\phi_{1}=0.4 \mathrm{rad}\right)$

\begin{tabular}{cccccccccc}
\hline$n_{i}$ & 1 & 2 & 3 & 4 & & 37 & 38 & 39 & 40 \\
\hline$\phi_{2}(\mathrm{rad})$ & 0.4103 & 0.4103 & 0.4103 & 0.4103 & $\ldots$ & 0.4103 & 0.4103 & 0.4103 & 0.4103 \\
$\alpha_{i}(\mathrm{rad})$ & 0.1468 & 0.3039 & 0.4610 & 0.6181 & $\ldots$ & 5.8017 & 5.9588 & 6.1158 & 6.2729 \\
$\beta_{i}(\mathrm{rad})$ & 4.0868 & 4.0848 & 4.0581 & 4.0171 & $\ldots$ & 2.8455 & -2.7910 & -2.3953 & -2.2427 \\
$\Delta_{\max }$ & -0.0042 & -0.0036 & -0.0036 & 0.0036 & $\ldots$ & -0.0195 & 0.1990 & 0.0711 & -0.0853 \\
$\times 10^{-12}$ & & & & & & & & & \\
\hline
\end{tabular}

\section{Meshing Point Position of Modified Cycloidal-pin Wheel}

The inevitable errors of dimensional chains, manufactures, installations of standard cycloidal-pin wheel cannot be compensated because of the multi teeth meshing without clearance. Hence the cycloid tooth 
profile must be modified in order to compensate the meshing backlash, which can compensate the manufacture and installation errors. The reasonable meshing backlash is guaranteed to fill lubricant and convenient assembly and disassembly. The modified cycloid-pin wheel includes equidistance modification $\Delta r_{r p}=0.01 \mathrm{~mm}$ and distance modification $\Delta r_{p}=-0.015 \mathrm{~mm}$ in this text. A modified cycloidal-pin wheel drive is shown in Fig. 3.The black solid line is the initial position of the pin wheel, the red dashed line is the initial meshing position of the modified cycloid-pin wheel after pin wheel rotated an angle. This angle is the minimum relative geometric angle $\gamma_{\min }$.

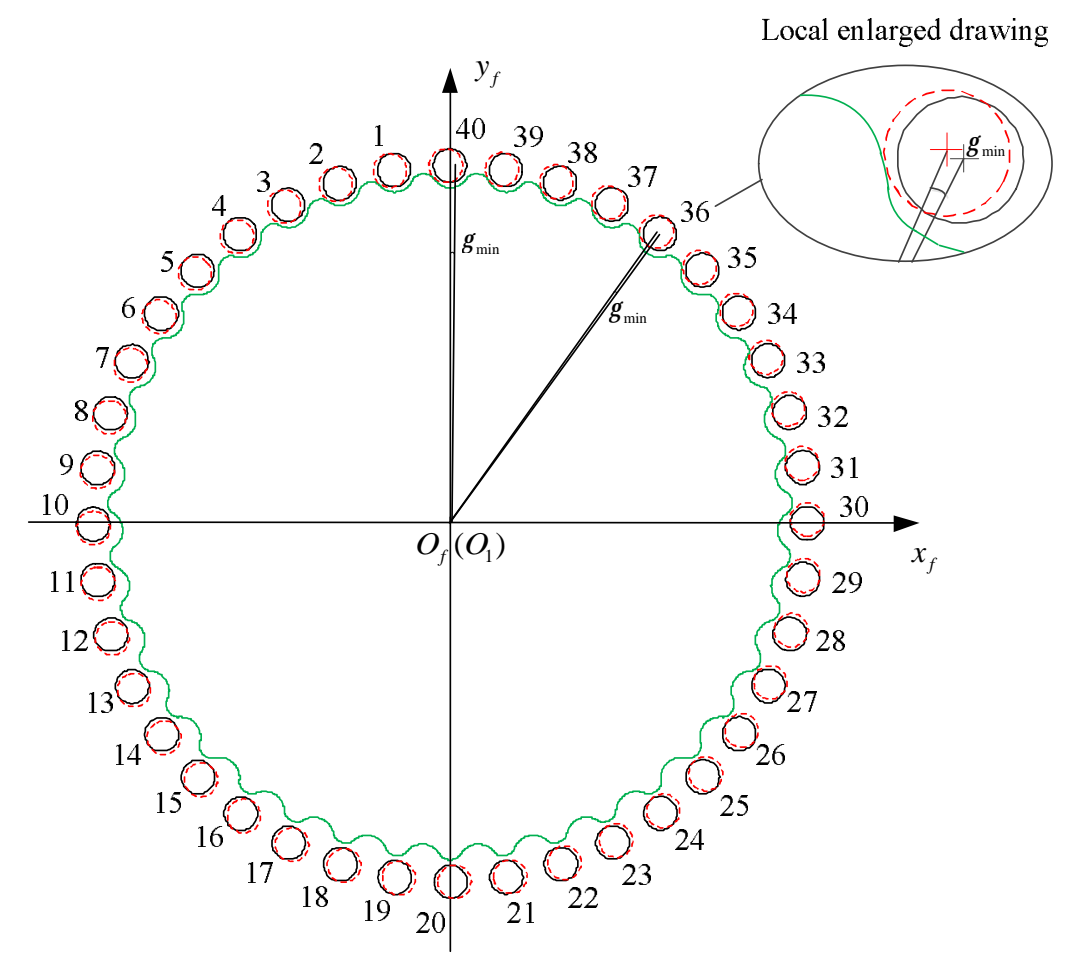

Fig. 3. Modified cycloid-pin wheel drive

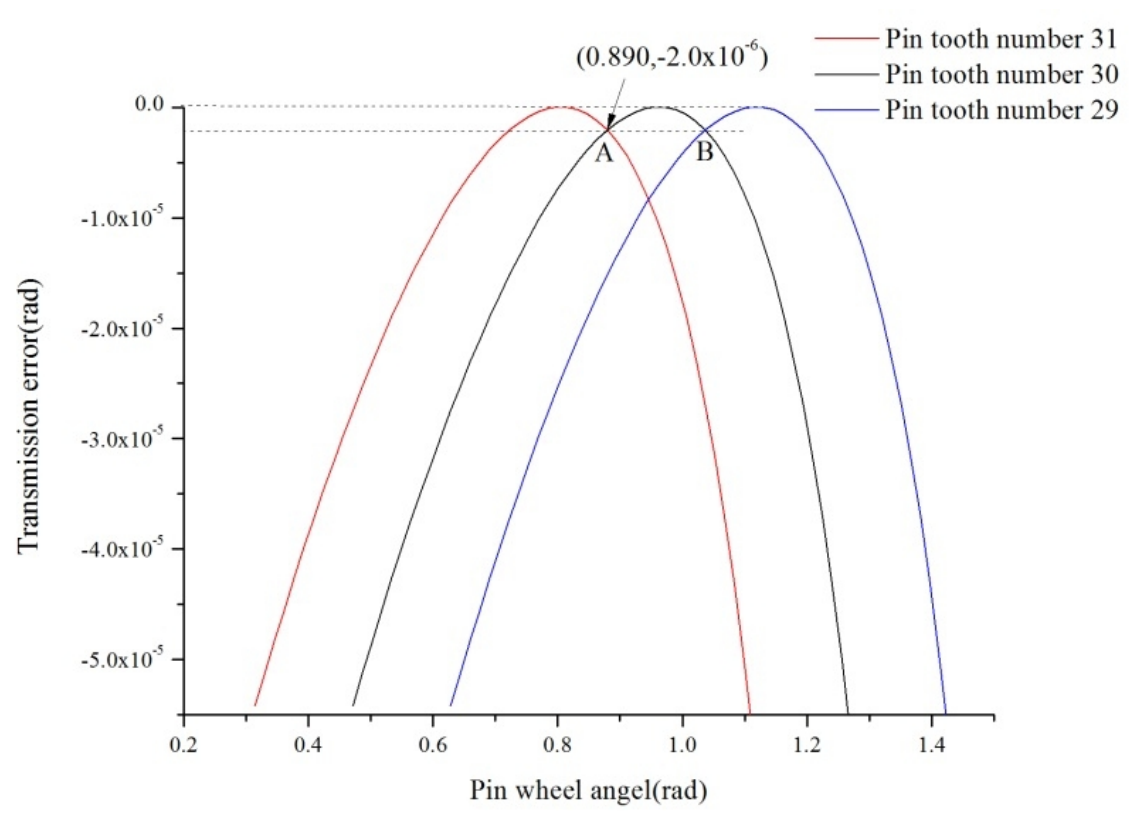

Fig. 4. Partial integrated transmission error curve

First, $\gamma_{\text {min }}$ is solved by TCA and the initial meshing pin tooth number $m$ is the corresponding pin tooth number. Second, the working points of the initial meshing pin tooth in the transmission process are obtained. In the pin wheel angle interval $\phi_{1}=\left[\gamma_{\min }, \gamma_{\min }+2 \pi / z_{p}\right]$, a certain step length $\Delta \phi_{1}$ is given, 
then the position parameters of each meshing point $\left(\phi_{2}, \alpha, \beta\right)$ are obtained. The appropriate reference point $\left(\phi_{10}, \phi_{20}\right)$ is selected and the transmission error curve at the $m$-th tooth is drawn by Eq. 4 . Because the engagement state of each pin tooth and cycloid gear is the same, the integrated transmission error curve of the modified cycloidal-pin could be obtained. As shown in Fig. 4, The area between A and B is the meshing working interval between the 30th pin tooth and the cycloid gear. Third, the only $\phi_{2}$ could be gotten by the integrated transmission error curve, then $(m, \alpha, \beta)$ also could be solved by TCA equations. Consequently, the meshing point position of the modified cycloidal-pin wheel at any angle would be solved, as shown in Table 2.In addition, the result also proves the theory that only one pair of teeth would mesh in the transmission process of modified cycloidal-pin wheel at any moment under no load.

$$
\Delta(\theta)=\left(\phi_{2}-\phi_{20}\right)-\left(\phi_{1}-\phi_{10}\right) \times z_{p} / z_{c}
$$

Table 2 Meshing point position of modified cycloidal-pin wheel

\begin{tabular}{cccccccccc}
\hline$\phi_{1}$ & 0 & $0.18 \pi$ & $0.36 \pi$ & $0.54 \pi$ & $\ldots$ & $1.26 \pi$ & $1.44 \pi$ & $1.62 \pi$ & $1.80 \pi$ \\
\hline$\phi_{2}$ & -0.0004 & 0.5796 & 1.1596 & 1.7396 & $\ldots$ & 4.0595 & 4.6395 & 5.2195 & 5.7995 \\
$m$ & 36 & 33 & 29 & 25 & $\ldots$ & 11 & 7 & 4 & 40 \\
$\alpha_{i}$ & 5.6555 & 5.1699 & 4.5270 & 3.8841 & $\ldots$ & 1.6271 & 0.9842 & 0.4986 & 6.1389 \\
$\beta_{i}$ & 2.1939 & 2.1958 & 2.1932 & 2.1957 & $\ldots$ & 2.1932 & 2.1957 & 2.1938 & 2.1932 \\
\hline
\end{tabular}

\section{Conclusions}

This paper focuses on calculating the meshing point position of cycloidal-pin wheel drive process in RV reducer. Ultimately, the meshing point position of the standard cycloidal-pin wheel and the modified cycloidal-pin wheel at any angle in the no-load condition have been solved by means of tooth contact analysis(TCA).In addition, the results prove that in the no-load condition all pairs of standard cycloidal-pin teeth would mesh and only one pair of modified cycloidal-pin tooth would mesh.

\section{Acknowledgements}

This work was financially supported by the National Natural Science Foundation (U1504522), Key Scientific and Technological Project in Henan Province (152102210075) and Major Scientific and Technological Project in Henan Province (161100211200).We would like to thank editor and anonymous reviewers for their constructive comments and helpful suggestions for improving the manuscript.

\section{References}

[1] Lixing Li, Chunhe Hong: Journal of Dalian Jiaotong University Vol. 13(1992)No. 1,p. 7-11.

[2] Wan-Sung Lin, Yi-Pei Shih and Jyh-Jone Lee:Mechanism \& Machine Theory Vol. 79(2014)No. 2, p. 184-197.

[3] Xiaozhong Deng, Yanzhen Zhang and Tianxing Li, etc:Journal of Mechanical Transmission Vol. 39(2015)No. 2, p. 162-165.

[4] Weidong He, Lijun Shan: Journal of Dalian Jiaotong University Vol. 37(2016)No. 5,p. 13-18.

[5] Dongsheng Zhang, Tianmin Guan:Journal of Machine Design Vol. 23(2006)No. 12,p. 28-31. 